\title{
Fractional neutral stochastic differential equations driven by $\alpha$-stable process
}

\section{Zhi Li}

School of Information and Mathematics, Yangtze University, Jingzhou 434023, China.

Communicated by D. Baleanu

\begin{abstract}
In this paper, we are concerned with a class of fractional neutral stochastic partial differential equations driven by $\alpha$-stable process. By the stochastic analysis technique, the properties of operator semigroup and combining the Banach fixed-point theorem, we prove the existence and uniqueness of the mild solutions to this kind of equations driven by $\alpha$-stable process. In the end, an example is given to demonstrate the theory of our work. (C)2017 All rights reserved.
\end{abstract}

Keywords: Fractional neutral SDEs, $\alpha$-stable process, existence and uniqueness.

2010 MSC: 60H15, 60G15, 60H05.

\section{Introduction}

In recent years, fractional calculus and fractional differential equations have attracted the attention of many researchers due to their important applications to problems in mathematical physics, chemistry, biology and engineering. Many results on existence and stability of solutions to various type of fractional differential equations have been obtained. For more details on this topic, one can refer to [2, 9, 16, 29].

The deterministic models often fluctuate due to noise. Systems are often subjected to random perturbations. Stochastic differential equations have been investigated by many authors due to playing a very important role in formulation and analysis of many phenomena in economic and finance, physics, mechanics, electric and control engineering, see, for example, Da Prato and Zabczyk [5], Liu [15], Luo and Liu [17], Jahanipur [8], and references therein. Subsequently, with the help of semigroup theory and fractional calculus technique, some authors have also considered fractional stochastic differential equations driven by Brownian motion. One can refer to the literatures [4, 10-13, 21, 22].

On the other hand, many researchers show the widespread interest in the topic of stochastic differential equations driven by $\alpha$-stable processes owing to the fact that the $\alpha$-stable noise exhibits the heavy tails, which have plenty of applications to problems in mathematical physics, chemistry, biology and engineering. For example, Priola and Zabczyk [20] gave a proper starting point on the investigation of structural properties of SPDEs driven by an additive cylindrical stable noise, Dong et al. [6] studied the invariant measures of stochastic 2D Navier-Stokes equation driven by $\alpha$-stable processes, $\mathrm{Xu}$ [25] studied the ergodicity of the stochastic real Ginzburg-Landau equation driven by $\alpha$-stable noise, Zhang [27]

Email address: lizhi_csu@126.com (Zhi Li)

doi:10.22436/jnsa.010.09.14 
established the Bismut-Elworthy-Li derivative formula for stochastic differential equations driven by $\alpha$ stable noise, and Bao and Yuan [3] discussed strong convergence of exponential integrator scheme based on spatial and time discretization for neutral stochastic partial differential equations driven by $\alpha$-stable processes. However, to the best of our knowledge, there are no results on fractional stochastic differential equations driven by $\alpha$-stable processes due to the fact that these process only has finite $p$-th moment for $p \in(0, \alpha)$ and the usual stochastic evolution does not admit a stochastic differential, a fact which leads to some powerful tools such as Itô's formula or Burkholder-Davis-Gundy's inequality in stochastic calculus being unavailable. To close the gap, we will make the attempt to investigate the property of dynamics for fractional stochastic differential equations driven by $\alpha$-stable processes in this paper.

To this end, in this paper we will focus on the following neutral fractional stochastic partial differential equations with delay driven by $\alpha$-stable noise

$$
\begin{cases}{ }^{C} D_{t}^{q}[x(t)-g(t, x(t-\tau))]=[A x(t)+f(t, x(t-\tau))] d t+\sigma(t) d Z(t), & t \in[0, T], \\ x_{0}(\cdot)=\xi \in D([-\tau, 0], H), & t \in[-\tau, 0],\end{cases}
$$

where the fractional derivative ${ }^{C} D^{q}, q \in(1 / 2,1]$, is understood in the Caputo sense, $A$ is the infinitesimal generator of an analytic semigroup of bounded linear operators $\{S(t)\}_{t \geqslant 0}$ in a Hilbert space $H$ with inner product $\langle\cdot, \cdot\rangle$ and norm $\|\cdot\|, \mathrm{D}([-\tau, 0], \mathrm{H})$ is the space of all càdlág functions paths from $[-\tau, 0]$ into $\mathrm{H}$, and $g, f: H \rightarrow H, \sigma:[0,+\infty) \rightarrow \mathbb{R}^{+}$are given functions to be specified later. The aim of this paper is to investigate the existence and uniqueness of the mild solution to (1.1) by using the stochastic analysis techniques, the properties of operator semigroup and combining the fixed point theorem.

The rest of this paper is organized as follows. In Section 2, we introduce some necessary notations and preliminaries. In Section 3, we devote to investigating the existence and uniqueness of the mild solutions to (1.1). In Section 4, we give an example to illustrate the efficiency of the obtained result.

\section{Preliminary}

In this section we collect some notions, conceptions and lemmas on $\alpha$-stable process and recall some basic results which will be used throughout the whole of this paper.

Let $Z(t)$ be a cylindrical $\alpha$-stable process, $\alpha \in(0,2)$, defined by

$$
Z(t):=\sum_{m=1}^{\infty} \beta_{m} Z_{m}(t) e_{m} .
$$

Here $\left\{\boldsymbol{e}_{\mathrm{m}}\right\}_{\mathrm{m} \geqslant 1}$ is an orthonormal basis of $\mathrm{H},\left\{\mathrm{Z}_{\mathrm{m}}(\mathrm{t})\right\}_{\mathrm{m} \geqslant 1}$ are independent, real-valued, normalized, symmetric $\alpha$-stable Lévy processes defined on stochastic basis $\left(\Omega, \mathcal{F},\left\{\mathcal{F}_{t}\right\}_{t \geqslant 0}, \mathbb{P}\right)$, and $\left\{\beta_{m}\right\}_{m} \geqslant 1$ is a sequence of positive numbers. Recall that a stochastic process $\left\{Z_{\alpha, \beta}(t): t \geqslant 0\right\}$ is called an $\alpha$-stable Lévy process if

(i) $Z_{\alpha, \beta}(0)=0$ a.s.;

(ii) $Z_{\alpha, \beta}$ has independent increments;

(iii) $Z_{\alpha, \beta}(t)-Z_{\alpha, \beta}(s) \sim \eta$ for any $0 \leqslant s<t<\infty$,

where $\eta$ stands for an $\alpha$-stable random variable, which is uniquely determined by its characteristic function involving four parameters: $\alpha \in(0,2]$, the index of stability; $\beta \in[-1,1]$, the skewness parameter; $\sigma \in(0, \infty)$, the scale parameter; $\mu \in(-\infty, \infty)$ the shift, which has the form

$$
\phi_{\eta}(u)=\mathbb{E} \exp (i u \eta)=\exp \left\{-\sigma^{\alpha}|u|^{\alpha}(1-i \beta \operatorname{sgn}(u) \Phi)+i \mu u\right\}, \quad u \in \mathbb{R},
$$

where $\Phi=\tan (\pi \alpha / 2)$ for $\alpha \neq 1$ and $\Phi=-(2 / \pi) \log |u|$ for $\alpha=1$. We call $\eta$ is strictly $\alpha$-stable whenever $\mu=0$, and if, in addition, $\beta=0, \eta$ is said to be symmetric $\alpha$-stable. For a real-valued normalized (standard) symmetric $\alpha$-stable Lévy process $z(t), \alpha \in(0,2)$, it has the characteristic function

$$
\operatorname{Eexp}(\operatorname{iuz}(\mathrm{t}))=\mathrm{e}^{-\mathrm{t}|\mathrm{u}|^{\alpha}}, \quad u \in \mathbb{R},
$$

and the Lévy measure $\lambda_{\alpha}(d x):=\frac{c_{\alpha}}{|x|^{1+\alpha}}, x \in \mathbb{R}-0$, where $c_{\alpha}$ is some constant. For more details of $\alpha$-stable processes, we can refer to [1] and [24]. 
Let $A$ be the infinitesimal generator of an analytic semigroup $(S(t))_{t \geqslant 0}$ and $0 \in \rho(A)$, where $\rho(A)$ is the resolvent set of $A$, then it is possible to define the fractional power $(-A)^{k}$ for $0<k \leqslant 1$, as a closed linear operator on its domain $D(-A)^{k}$. Furthermore, the subspace $D(-A)^{k}$ is dense in $H$, and the expression

$$
\|h\|_{k}=\left\|(-A)^{k} h\right\|
$$

defines a norm in $D(-A)^{k}$. If $H_{k}$ represents the space $D(-A)^{k}$ endowed with the norm $\|\cdot\|_{k}$, then the following properties are well-known (cf. [19, Theorem 6.13 p.74]).

Lemma 2.1. Suppose that the previous conditions are satisfied.

(1) Let $0<\mathrm{k} \leqslant 1$. Then $\mathrm{H}_{\mathrm{k}}$ is a Banach space.

(2) If $0<\mathrm{k} \leqslant \mathrm{l}$ then the injection $\mathrm{H}_{\mathrm{l}} \hookrightarrow \mathrm{H}_{\mathrm{k}}$ is continuous.

(3) For every $0<k \leqslant 1$ there exists $M_{k}>0$ such that

$$
\left\|(-A)^{k} S(t)\right\| \leqslant M_{k} t^{-k} e^{-\lambda t}, t>0, \lambda>0 .
$$

Now, we recall some notations and preliminary results about fractional calculus and some special functions.

Definition 2.2. The Riemann-Liouville fractional integral of the order $q>0$ of $f:[0, T] \rightarrow H$ is defined by

$$
J_{t}^{q} f(t)=\frac{1}{\Gamma(q)} \int_{0}^{t}(t-s)^{q-1} f(s) d s,
$$

where $\Gamma(\cdot)$ is the standard Gamma function.

Definition 2.3. The Riemann-Liouville fractional derivative of the order $q \in(0,1]$ of $f:[0, T] \rightarrow H$ is defined by

$$
D_{t}^{q} f(t)=\frac{d}{d t} J_{t}^{1-q} f(t)
$$

Definition 2.4. The Caputo fractional derivative of the order $q \in(0,1]$ of $f:[0, T] \rightarrow H$ is defined by

$$
{ }^{C} D_{t}^{q} f(t)=D_{t}^{q}(f(t)-f(0)) .
$$

The Laplace transform of Caputo fractional derivative is given by

$$
L\left\{{ }^{C} D_{t}^{q} u(t)\right\}=\lambda^{q} \hat{u}(\lambda)-\lambda^{q-1} u(0),
$$

where $\hat{u}(\lambda)$ is the Laplace transform of $u$ defined by

$$
\hat{u}(\lambda)=\int_{0}^{\infty} e^{-\lambda t} u(t) d t, \quad \Re \lambda>\omega,
$$

where $\Re \lambda$ stands for the real part of the complex number $\lambda$.

Definition 2.5. The Mittag-Leffler function is defined by

$$
\mathrm{E}_{\mathrm{q}, \mathrm{p}}(z)=\sum_{n=0}^{\infty} \frac{z^{\mathrm{n}}}{\Gamma(\mathrm{qn}+\mathrm{p})}, \mathrm{p}, \mathrm{q}>0, z \in \mathbb{C} .
$$

When $\mathrm{p}=1$, set $\mathrm{E}_{\mathrm{q}}(z)=\mathrm{E}_{\mathrm{q}, 1}(z)$.

Definition 2.6. The Mainardi's function is defined by

$$
M_{q}(z)=\sum_{n=0}^{\infty} \frac{(-z)^{n}}{n ! \Gamma(-q n+1-q)}, 0<q<1, z \in \mathbb{C} .
$$


The Laplace transform of Mainardi's function $M_{q}(r)$ is (see [18]):

$$
\int_{0}^{\infty} e^{-\lambda r} M_{q}(r) d r=E_{q}(-\lambda)
$$

By (2.2) and (2.3), it is clear that

$$
\int_{0}^{\infty} M_{\mathrm{q}}(\mathrm{r}) \mathrm{dr}=1,0<\mathrm{q}<1
$$

On the other hand, $M_{q}(z)$ satisfies the following equality (see [18])

$$
\int_{0}^{\infty} \frac{q}{r^{q+1}} M_{q}\left(1 / r^{q}\right) e^{-\lambda r} d r=e^{-\lambda^{q}}
$$

and the equality (see [18])

$$
\int_{0}^{\infty} r^{\delta} M_{q}(r) d r=\frac{\Gamma(\delta+1)}{\Gamma(q \delta+1)}, \delta>-1,0<q<1 .
$$

Throughout this paper we impose the following assumptions.

(H1) The operator $(A, D(A))$ is a self-adjoint operator on the separable Hilbert space $\mathrm{H}$ admitting a discrete spectrum

$$
0 \leqslant \lambda_{1} \leqslant \lambda_{2} \leqslant \cdots \leqslant \lambda_{m} \leqslant \cdots \leqslant \lim _{m \rightarrow \infty} \lambda_{m}=\infty
$$

with corresponding eigenbasis $\left\{\boldsymbol{e}_{\mathrm{m}}\right\}_{\mathrm{m} \geqslant 1}$ of $\mathrm{H}$ and generating an analytic semigroup $\mathrm{S}(\mathrm{t})=e^{\mathrm{t} A}$, $t \geqslant 0,0 \in \rho(A)$, such that $\left\|e^{t A}\right\| \leqslant M e^{-\lambda_{1} t}$.

(H2) There exists a positive constant $K_{1}$ such that for all $x, y \in H$ and $t \geqslant 0$,

$$
\|f(t, x)-f(t, y)\| \leqslant K_{1}\|x-y\|, \quad\|f(t, x)\| \leqslant K_{1}(1+\|x\|) .
$$

(H3) There exists $k \in(0,1]$ and a positive constant $K_{2}$ such that for all $x, y \in H$ and $t \geqslant 0$,

$$
\left\|(-A)^{k} g(t, x)-(-A)^{k} g(t, y)\right\| \leqslant K_{2}\|x-y\|, \quad g(t, 0)=0, \quad 5 M^{p}\left\|(-A)^{-k}\right\|^{p} K_{2}^{p}<1 .
$$

(H4) There exists a constant $\lambda>\frac{1}{1+\alpha q-\alpha}$ such that the function $\sigma:[0,+\infty) \rightarrow \mathbb{R}^{+}$satisfies

$$
\int_{0}^{T} \sigma^{\alpha \lambda}(s) d s<\infty
$$

\section{Existence and uniqueness}

In this section, we shall prove the existence and uniqueness of the mild solution to equation (1.1). For $0<\mathrm{q}<1$, set $\mathrm{T}_{\mathrm{q}}(\mathrm{t}) x=\int_{0}^{\infty} M_{\mathrm{q}}(\mathrm{r}) S\left(\mathrm{t}^{\mathrm{q}} \mathrm{r}\right) x \mathrm{dr}$ and $S_{\mathrm{q}}(\mathrm{t}) x=\int_{0}^{\infty} q r M_{\mathrm{q}}(\mathrm{r}) S\left(\mathrm{t}^{\mathrm{q}} \mathrm{r}\right) x \mathrm{dr}, \mathrm{t} \geqslant 0, x \in X$. It is known that $u(t)=T_{q}(t) u_{0}+\int_{0}^{t}(t-s)^{q-1} S_{q}(t-s) f(s, u(s)) d s$, and is the mild solution to the deterministic fractional equation

$$
\left\{\begin{array}{l}
{ }^{C} D_{t}^{q} u(t)=A(t) u(t)+f(t, u(t)) d t, t \in[0, b] \\
u(0)=u_{0}
\end{array}\right.
$$

see, for example, $[14,28]$. Motivated by this result and noting Definitions 2.4 and 2.5, we present the following definition of mild solutions to (1.1).

Definition 3.1. An $\mathcal{F}_{t}$-adapted càdlág stochastic process $x(t), t \in[-\tau, T]$ is called the mild solution for (1.1) if 
(i) $x_{0}=\xi \in \mathrm{D}([-\tau, 0] ; \mathrm{H})$;

(ii) for arbitrary $t \in[0, T], x(t)$ satisfies the following integral equation:

$$
\begin{aligned}
x(t)= & T_{q}(t)[\xi(0)+g(0, \xi(-\tau))]+g(t, x(t-\tau))+\int_{0}^{t}(t-s)^{q-1} A S_{q}(t-s) g(s, x(s-\tau)) d s \\
& +\int_{0}^{t}(t-s)^{q-1} S_{q}(t-s) f(s, x(s-\tau)) d s+\int_{0}^{t}(t-s)^{q-1} S_{q}(t-s) \sigma(s) d Z(s) .
\end{aligned}
$$

The following properties of $T_{q}(t)$ and $S_{q}(t)$ appeared in [29] are useful.

Lemma 3.2. Under the assumption (H1),

(i) for any fixed $\mathrm{t} \geqslant 0, \mathrm{~T}_{\mathrm{q}}(\mathrm{t})$ and $\mathrm{S}_{\mathrm{q}}(\mathrm{t})$ are linear and bounded operators such that for any $\mathrm{x} \in \mathrm{H},\left\|\mathrm{T}_{\alpha}(\mathrm{t}) \mathrm{x}\right\| \leqslant$ $M\|x\|,\left\|S_{\alpha}(t) x\right\| \leqslant \frac{M q}{\Gamma(1+q)}\|x\| ;$

(ii) $\mathrm{T}_{\mathrm{q}}(\mathrm{t})$ and $\mathrm{S}_{\mathrm{q}}(\mathrm{t})$ are strongly continuous;

(iii) for any $x \in H, \beta \in(0,1)$, and $k \in(0,1]$, we have

$$
\operatorname{AS}_{\mathrm{q}}(\mathrm{t}) x=A^{1-\beta} S_{\mathrm{q}}(\mathrm{t}) A^{\beta} x \text { and }\left\|A^{k} S_{\mathrm{q}}(\mathrm{t})\right\| \leqslant \frac{\mathrm{q} M_{\mathrm{k}}}{\mathrm{t}^{\mathrm{q} k}} \frac{\Gamma(2-\mathrm{k})}{\Gamma(1+\mathrm{q}(1-\mathrm{k}))} .
$$

Lemma 3.3. Let (H1) hold, then for any $t \geqslant 0$ and $p>0$

$$
\mathbb{E}\left\|\int_{0}^{t}(t-s)^{q-1} S_{q}(t-s) \sigma(s) d Z(s)\right\|^{p} \leqslant C_{p, \alpha}\left(\sum_{k=1}^{\infty} \beta_{k}^{\alpha} \int_{0}^{t}(t-s)^{\alpha q-\alpha} S_{q}^{\alpha}(t-s) \sigma^{\alpha}(s) d s\right)^{p / \alpha},
$$

where the constant $C_{p, \alpha}>0$ depends on $p$ and $\alpha$.

Proof. By virtue of (2.1), we can easily calculate that

$$
\int_{0}^{t}(t-s)^{q-1} S_{q}(t-s) \sigma(s) d Z(s)=\sum_{k=1}^{\infty} \Lambda_{k}(t) e_{k},
$$

where $\Lambda_{k}(t):=\int_{0}^{t} \beta_{k}(t-s)^{q-1} S_{q}(t-s) \sigma(s) d Z_{k}(s)$. Let $\left\{r_{k}\right\}_{k} \geqslant 1$ be a Rademacher sequence defined on a new probability space $\left(\Omega^{\prime}, \mathcal{F}^{\prime},\left\{\mathcal{F}_{t}^{\prime}\right\}_{t} \geqslant 0, \mathbb{P}^{\prime}\right)$, i.e., $r_{k}: \Omega^{\prime} \rightarrow\{1,-1\}$ are i.i.d. with $\mathbb{P}^{\prime}\left(r_{k}=1\right)=\mathbb{P}^{\prime}\left(r_{k}=\right.$ $-1)=1 / 2$.

The following Khintchine's inequality holds, for arbitrary real numbers $c_{1}, \cdots, c_{n}$, for any $p>0$

$$
\left(\sum_{k \geqslant 1} c_{k}^{2}\right)^{1 / 2} \leqslant M_{1}(p)\left(\mathbb{E}^{\prime}\left|\sum_{k \geqslant 1} r_{k} c_{k}\right|^{p}\right)^{1 / p}
$$

(see, for instance, [7]), where the constant $C_{p}$ depends only on $p$ (for $p=1$, we have $c_{1}=\sqrt{2}$ ) and $\mathbb{E}^{\prime}$ indicates the expectation with respect to $\mathbb{P}^{\prime}$.

Then, by (3.1), we can write

$$
\left(\sum_{k=1}^{\infty}\left(\int_{0}^{t}(t-s)^{q-1} S_{q}(t-s) \sigma(s) d Z_{k}(s)\right)^{2}\right)^{1 / 2} \leqslant M_{1}(p)\left(\mathbb{E}^{\prime}\left|\sum_{k \geqslant 1} r_{k} \Lambda_{k}(t)\right|^{p}\right)^{1 / p} .
$$

Using the Fubini theorem and the property of the $\alpha$-stable process $Z(t)$ (see [20]), we have

$$
\begin{aligned}
& \mathbb{E}\left\|\int_{0}^{t}(t-s)^{q-1} S_{q}(t-s) \sigma(s) d Z(s)\right\|^{p} \\
& \leqslant\left(M_{1}(p)\right)^{p} \mathbb{E}\left[\mathbb{E}^{\prime}\left|\sum_{k \geqslant 1} r_{k} \Lambda_{k}(t)\right|^{p}\right] \\
& =\left(M_{1}(p)\right)^{p} \mathbb{E}^{\prime}\left[\mathbb{E}\left|\sum_{k \geqslant 1} r_{k} \Lambda_{k}(t)\right|^{p}\right] \\
& =\left(M_{1}(p)\right)^{p} \mathbb{E}^{\prime}\left[\mathbb{E}\left|\sum_{k \geqslant 1} r_{k} \int_{0}^{t} \beta_{k}(t-s)^{q-1} S_{q}(t-s) \sigma(s) d Z_{k}(s)\right|^{p}\right] .
\end{aligned}
$$


Note that $\left|r_{k}\right|=1$ for any $k \geqslant 1$. Then by using properties of $\alpha$-stable processes again, it is not difficult to get for any $t \geqslant 0, \lambda \in \mathbb{R}$ that

$$
\mathbb{E} \exp \left[i \lambda \sum_{k=1}^{\infty} r_{k} \Lambda_{k}(t)\right]=\exp \left[-|\lambda|^{\alpha} \sum_{k=1}^{\infty} \beta_{k}^{\alpha} \int_{0}^{t}(t-s)^{\alpha q-\alpha} S_{q}^{\alpha}(t-s) \sigma^{\alpha}(s) d s\right] .
$$

Recall the fact (see page 18, [23]) that if $\mathrm{Y}$ is a symmetric random variable satisfying

$$
\mathbb{E}\left[e^{i \lambda Y}\right]=e^{-\sigma^{\alpha}|\lambda|^{\alpha}}, \quad \sigma \in \mathbb{R}
$$

for some $\alpha \in(0,2)$ and any $\lambda \in \mathbb{R}$, then for all $p \in(0, \alpha)$,

$$
\mathbb{E}|Y|^{p}=M_{2}(\alpha, p) \sigma^{p}
$$

where $M_{2}(\alpha, p)>0$ is a constant depending only on $\alpha$ and $p$. Applying this result to (3.2) and (3.3), we then obtain that

$$
\mathbb{E}\left\|\int_{0}^{t}(t-s)^{q-1} S_{q}(t-s) \sigma(s) d Z(s)\right\|^{p} \leqslant C_{p, \alpha}\left(\sum_{k=1}^{\infty} \beta_{k}^{\alpha} \int_{0}^{t}(t-s)^{\alpha q-\alpha} S_{q}^{\alpha}(t-s) \sigma^{\alpha}(s) d s\right)^{p / \alpha},
$$

where the constant $C_{p, \alpha}=\left(M_{1}(p)\right)^{p} M_{2}(\alpha, p)>0$ depends on $p$ and $\alpha$. The proof is thus complete.

Theorem 3.4. Assume that (H1)-(H4) hold, and

$$
C:=\sum_{k=1}^{\infty} \beta_{k}^{\alpha}<\infty
$$

holds for $\alpha \in(1,2), \mathrm{q} \in(1 / 2,1)$. Then (1.1) has a unique mild solution on $[-\tau, \mathrm{T}]$.

Proof. Fix $\mathrm{T}>0$ and denote by $S_{\mathrm{T}}$ the Banach space of all càdlág $\mathrm{H}$-valued processes $x(t) \in \mathrm{D}([-\tau, \mathrm{T}] ; \mathrm{H})$ with initial data $x(t)=\xi(t)$ for $t \in[-\tau, 0]$ equipped with the supremum norm

$$
\|x(t)\|_{S_{T}}=\sup _{-\tau \leqslant t \leqslant T}\left(\mathbb{E}\|x(t)\|^{p}\right)^{1 / p} .
$$

Define the operator $\Psi$ on $S_{T}$ by $\Psi(x)(t)=\xi(t)$ for $t \in[-\tau, 0]$ and for $t \in[0, T]$

$$
\begin{aligned}
\Psi(x)(t)= & T_{q}(t)[\xi(0)+g(0, \xi(-\tau))]+g(t, x(t-\tau))+\int_{0}^{t}(t-s)^{q-1} A S_{q}(t-s) g(s, x(s-\tau)) d s \\
& +\int_{0}^{t}(t-s)^{q-1} S_{q}(t-s) f(s, x(s-\tau)) d s+\int_{0}^{t}(t-s)^{q-1} S_{q}(t-s) \sigma(s) d Z(s) .
\end{aligned}
$$

Then it is clear that to prove the existence of mild solutions to (1.1) is equivalent to find a fixed point for the operator $\Psi$. Next we will show by using the Banach fixed point theorem that $\Psi$ has a unique fixed point. We divide the subsequent proof into two steps.

Step 1. We show that $\Psi(x)(t) \subset S_{T}$ for $t \in[-\tau, T]$. It is trivial for the case $t \in[-\tau, 0]$. For $t \in[0, T]$, and for any fixed $x \in S_{T}$, using the essential inequality: $\left(\sum_{i=1}^{n} a_{i}\right)^{p} \leqslant C_{r}\left(\sum_{i=1}^{n} a_{i}^{p}\right)$, here $C_{r}=1$ when $p \leqslant 1$, $\mathrm{C}_{\mathrm{r}}=\mathrm{n}^{\mathrm{p}-1}$ when $\mathrm{p}>1$, we have

$$
\begin{aligned}
\mathbb{E}\|\Psi(x)(t)\|^{p} \leqslant & 5^{p-1} \mathbb{E}\left\|T_{q}(t)[\xi(0)+g(0, \xi(-\tau))]\right\|^{p}+5^{p-1} \mathbb{E}\|g(t, x(t-\tau))\|^{p} \\
& +5^{p-1} \mathbb{E}\left\|\int_{0}^{t} A S_{q}(t-s)(t-s)^{q-1} g(s, x(s-\tau)) d s\right\|^{p} \\
& +5^{p-1} \mathbb{E}\left\|\int_{0}^{t} S_{q}(t-s)(t-s)^{q-1} f(s, x(s-\tau)) d s\right\|^{p} \\
& +5^{p-1} \mathbb{E}\left\|\int_{0}^{t} S_{q}(t-s)(t-s)^{q-1} \sigma(s) d Z(s)\right\|^{p}:=\sum_{i=1}^{5} I_{i} .
\end{aligned}
$$


It follows from $(\mathrm{H} 1),(\mathrm{H} 3)$, and Lemma 3.2 that

$$
\begin{aligned}
\mathrm{I}_{1} & =5^{\mathrm{p}-1} \mathbb{E}\left\|(-\mathrm{A})^{-\mathrm{k}} \mathrm{T}_{\mathrm{q}}(\mathrm{t})(-\mathrm{A})^{\mathrm{k}}[\xi(0)+\mathrm{g}(0, \xi(-\tau))]\right\|^{\mathrm{p}} \\
& \leqslant 10^{\mathrm{p}-1} M^{\mathrm{p}} \mathbb{E}\|\xi(0)\|^{\mathrm{p}}+10^{\mathfrak{p}-1} \mathrm{M}^{\mathrm{p}} \mathrm{K}_{2}^{\mathrm{p}}\left\|(-\mathrm{A})^{-\mathrm{k}}\right\|^{\mathrm{p}} \mathbb{E}\|\xi(-\tau)\|^{\mathrm{p}} \\
& \leqslant 10^{\mathrm{p}-1} M^{\mathrm{p}}\left(1+\mathrm{K}_{2}^{\mathrm{p}}\left\|(-\mathrm{A})^{-\mathrm{k}}\right\|^{\mathrm{p}}\right) \sup _{-\tau \leqslant s \leqslant 0} \mathbb{E}\|\xi(\mathrm{s})\|^{\mathrm{p}} .
\end{aligned}
$$

For the second term, $\mathrm{I}_{2}$, using the assumption (H3) again, we have

$$
\left.I_{2}=5^{p-1} \mathbb{E} \|(-A)^{-k}(-A)^{k} g(t, x(t-\tau))\right]\left\|^{p} \leqslant 5^{p-1} M^{p} K_{2}^{p}\right\|(-A)^{-k}\left\|^{p} \mathbb{E}\right\| x(t-\tau) \|^{p} .
$$

From (H3), the Hölder's inequality and Lemma 3.2, we can obtain

$$
\begin{aligned}
I_{3} & =5^{p-1} \mathbb{E}\left\|\int_{0}^{t}(-A)^{1-k} S_{q}(t-s)(t-s)^{q-1}(-A)^{k} g(s, x(s-\tau)) d s\right\|^{p} \\
& \leqslant 5^{p-1}\left|\frac{K_{2} q M_{1-k} \Gamma(2-k)}{\Gamma(1+q(1-k))}\right|^{p} \mathbb{E}\left(\int_{0}^{t}(t-s)^{q k-1}\|x(s-\tau)\| d s\right)^{p} \\
& \leqslant 5^{p-1}\left|\frac{K_{2} q M_{1-k} \Gamma(2-k)}{\Gamma(1+q(1-k))}\right|^{p}\left(\int_{0}^{t}(t-s)^{q k-1} d s\right)^{p-1} \int_{0}^{t}(t-s)^{q k-1} \mathbb{E}\|x(s-\tau)\|^{p} d s \\
& \leqslant 5^{p-1}\left|\frac{K_{2} q M_{1-k} \Gamma(2-k) t^{q k}}{\Gamma(1+q(1-k)) q k}\right|^{p} \sup _{-\tau \leqslant s \leqslant t} \mathbb{E}\|x(s)\|^{p} .
\end{aligned}
$$

By using Lemma 3.2, (H2), and the Hölder's inequality, we have

$$
\begin{aligned}
\mathrm{I}_{4} & =5^{p-1} \mathbb{E}\left\|\int_{0}^{\mathrm{t}} S_{\mathrm{q}}(\mathrm{t}-\mathrm{s})(\mathrm{t}-\mathrm{s})^{\mathrm{q}-1} f(s, x(s-\tau)) d s\right\|^{p} \\
& \leqslant 5^{p-1}\left|\frac{\mathrm{K}_{1} \mathrm{qM}}{\Gamma(1+\mathrm{q})}\right|^{p} \sup _{0 \leqslant t \leqslant T} \mathbb{E}\left(\int_{0}^{t}(t-s)^{q-1}(1+\|x(s-\tau)\|) d s\right)^{p} \\
& \leqslant 10^{p-1}\left|\frac{K_{1} M t}{\Gamma(1+q)}\right|^{p}+10^{p-1}\left|\frac{K_{1} M q}{\Gamma(1+q)}\right|^{p}\left(\int_{0}^{t}(t-s)^{q-1} d s\right)^{p-1} \int_{0}^{t}(t-s)^{q-1} \mathbb{E}\|x(s-\tau)\|^{p} d s \\
& \leqslant 10^{p-1}\left|\frac{K_{1} M t}{\Gamma(1+q)}\right|^{p}+10^{p-1}\left|\frac{K_{1} M t}{\Gamma(1+q)}\right|^{p} \sup _{-\tau \leqslant s \leqslant t} \mathbb{E}\|x(s)\|^{p} .
\end{aligned}
$$

By Lemma 3.3, we know that

$$
\begin{aligned}
I_{5} & \leqslant C_{p, \alpha}\left(\sum_{k=1}^{\infty} \beta_{k}^{\alpha} \int_{0}^{t}\left(S_{q}(t-s)\right)^{\alpha}(t-s)^{\alpha(q-1)} \sigma^{\alpha}(s) d s\right)^{p / \alpha} \\
& \leqslant C_{p, \alpha}\left|\frac{M q}{\Gamma(1+q)}\right|^{p}\left(\sum_{k=1}^{\infty} \beta_{k}^{\alpha} \int_{0}^{t}(t-s)^{\alpha(q-1)} \sigma^{\alpha}(s) d s\right)^{p / \alpha}
\end{aligned}
$$

Notice that $\alpha \in(1,2)$ and $q \in(1 / 2,1]$, then $\lambda>\frac{1}{1+\alpha q-\alpha}>1$. So we can obtain by the Hölder's inequality that

$$
\begin{aligned}
I_{5} & \leqslant C_{p, \alpha}\left|\frac{M q}{\Gamma(1+q)}\right|^{p}\left[\sum_{k=1}^{\infty} \beta_{k}^{\alpha}\left(\int_{0}^{t}(t-s)^{\frac{\lambda \alpha(q-1)}{\lambda-1}} d s\right)^{\frac{\lambda-1}{\lambda}} \cdot\left(\int_{0}^{t} \sigma^{\alpha \lambda}(s) d s\right)^{1 / \lambda}\right]^{p / \alpha} \\
& \leqslant C_{p, \alpha}\left|\frac{M q}{\Gamma(1+q)}\right|^{p} T^{\frac{(\alpha q-\alpha+1) \lambda-1}{\lambda}}\left(\int_{0}^{t} \sigma^{\alpha \lambda}(s) d s\right)^{\frac{p}{\alpha \lambda}}\left(\sum_{k=1}^{\infty} \beta_{k}^{\alpha}\right)^{p / \alpha} \\
& <\infty
\end{aligned}
$$


Thus, we derive from (3.5)-(3.10) that, for some constants $c_{1}, c_{2}$, and $c_{3}$,

$$
\sup _{0 \leqslant t \leqslant T} \mathbb{E}\|\Psi(x)(t)\|^{p} \leqslant c_{1}+c_{2} \sup _{-\tau \leqslant \theta \leqslant 0} \mathbb{E}\|\xi(\theta)\|^{p}+c_{3} \sup _{0 \leqslant t \leqslant T} \mathbb{E}\|x(t)\|^{p} .
$$

Hence, $\Psi(x)(t) \subset S_{T}$.

Step 2. We shall show that the mapping $\Psi$ is contractive. Let $x, y \in S_{T}$. For any fixed $t \in[0, T]$, we have

$$
\begin{aligned}
\mathbb{E}\|\Psi(x)(t)-\Psi(y)(t)\|^{p} \leqslant & 3^{p-1} \mathbb{E}\|g(t, x(t-\tau))-g(t, y(t-\tau))\|^{p} \\
& +3^{p-1} \mathbb{E}\left\|\int_{0}^{t} A S_{q}(t-s)(t-s)^{q-1}[g(s, x(s-\tau))-g(s, y(s-\tau))] d s\right\|^{p} \\
& +3^{p-1} \mathbb{E}\left\|\int_{0}^{t} S_{q}(t-s)(t-s)^{q-1}[f(s, x(s-\tau))-f(s, y(s-\tau))] d s\right\|^{p}
\end{aligned}
$$

By the assumptions (H2), (H3), and the Hölder's inequality, we obtain

$$
\begin{aligned}
\mathbb{E}\|\Psi(x)(t)-\Psi(y)(t)\|^{p} \leqslant & 3^{p-1} M^{p} K_{2}^{p}\left\|(-A)^{-k}\right\|^{p} \mathbb{E}\|x(t-\tau)-y(t-\tau)\|^{p} \\
& +3^{p-1}\left|\frac{K_{2} q M_{1-k} \Gamma(2-k) t^{q k}}{\Gamma(1+q(1-k)) q k}\right|^{p} \sup _{0 \leqslant s \leqslant t} \mathbb{E}\|x(s)-y(s)\|^{p} \\
& +3^{p-1}\left|\frac{K_{1} M t}{\Gamma(1+q)}\right|^{p} \sup _{0 \leqslant s \leqslant t} \mathbb{E}\|x(s)-y(s)\|^{p} .
\end{aligned}
$$

Then,

$$
\sup _{0 \leqslant t \leqslant T} \mathbb{E}\|\Psi(x)(t)-\Psi(y)(t)\|^{p} \leqslant 3^{p-1}\left(M^{p} K_{2}^{p}\left\|(-A)^{-k}\right\|^{p}+C_{1} T^{q k p}+C_{2} T^{p}\right) \sup _{0 \leqslant t \leqslant T} \mathbb{E}\|x(t)-y(t)\|^{p},
$$

where $x(t)=y(t)$ on $[-\tau, 0]$, and $C_{1}>0, C_{2}>0$ are two bounded constants. Hence, by the condition $5 M^{p} K_{2}^{p}\left\|(-A)^{-k}\right\|^{p}<1$, choosing sufficiently small $T_{1}$ such that

$$
3^{p-1}\left(M^{p} K_{2}^{p}\left\|(-A)^{-k}\right\|^{p}+C_{1} T^{q k p}+C_{2} T^{p}\right)<1,
$$

we can conclude that $\Psi$ is a contraction mapping on $S_{T_{1}}$ and therefore has a unique fixed point, which is a mild solution of (1.1) on $\left[0, \mathrm{~T}_{1}\right]$. This procedure can be repeated in order to extend the solution to the entire interval $[0, T]$ in finitely many steps. This completes the proof.

\section{An example}

In this section, an example is provided to illustrate the theory obtained.

Example 4.1. We consider the following neutral fractional stochastic partial functional differential equation driven by $\alpha$-stable process:

$$
\left.{ }^{C} D_{t}^{q}\left[x(t)-\int_{0}^{\pi} \varphi(-\tau, \zeta, x) u(t-\tau, \zeta) d \zeta\right)\right]=\left[\frac{\partial^{2}}{\partial x^{2}} u(t, x)+\phi(u(t-\tau), x)\right] d t+\sigma(t) d Z(t, x),
$$

with the Dirichlet boundary condition

$$
u(t, 0)=u(t, \pi)=0, \quad t \in[0, T],
$$

and the initial condition

$$
u(\theta, x)=\Psi(\theta, x), \quad \theta \in[-\tau, 0], x \in(0, \pi)
$$


Furthermore, let $\phi: \mathbb{R} \rightarrow \mathbb{R}$ be Lipschitzian. Assume further that $\varphi:[-\tau, 0] \times[0, \pi] \times[0, \pi] \rightarrow \mathbb{R}$ is measurable such that $\varphi(\cdot, \cdot, 0)=\varphi(\cdot, \cdot, \pi)=0$ and

$$
\mathrm{N}:=\int_{0}^{\pi} \int_{0}^{\pi}\left(\frac{\partial}{\partial x} \varphi(-\tau, \zeta, x)\right)^{2} \mathrm{~d} \zeta \mathrm{d} x<\infty
$$

Let $\mathrm{H}=\mathrm{L}^{2}(0, \pi)$ and $\mathrm{A}$ be given by

$$
A:=\frac{\partial^{2}}{\partial x^{2}}, \quad D(A):=H^{2}(0, \pi) \cap H_{0}^{1}(0, \pi),
$$

where $H^{k}(0, \pi), k=1,2$, represents the classical Sobolev spaces, and $H_{0}^{1}(0, \pi)$ is the subspace of $H^{1}(0, \pi)$ of all functions vanishing at 0 and $\pi$. Note that $A$ is a self-adjoint negative operator in $\mathrm{H}$ and $A e_{k}=-k^{2} e_{k}$ with $e_{k}(\xi)=(2 / \pi)^{1 / 2} \sin m \xi$ for $m \in \mathbb{N}$ and $\xi \in[0, \pi]$. We can easily know that $A$ is the infinitesimal generator of an analytic semigroup $S(t)=e^{t A}, t \geqslant 0$, in $H$ and the operator $(A, D(A))$ is a self-adjoint operator on the separable Hilbert space $\mathrm{H}$ admitting a discrete spectrum

$$
0 \leqslant \lambda_{1} \leqslant \lambda_{2} \leqslant \cdots \leqslant \lambda_{m} \leqslant \cdots \leqslant \lim _{m \rightarrow \infty} \lambda_{m}=\infty
$$

with $\lambda_{k}=k^{2}$ and $\|S(t)\| \leqslant e^{-t}$. This implies that (H1) holds. Furthermore, we know that

$$
(-A)^{-\frac{1}{2}} \zeta=\sum_{n=1}^{\infty} \frac{1}{n}\left\langle\zeta, e_{n}\right\rangle e_{n}, \quad \zeta \in H,
$$

and

$$
(-A)^{\frac{1}{2}} \zeta=\sum_{n=1}^{\infty} n\left\langle\zeta, e_{n}\right\rangle e_{n}, \quad \zeta \in D\left((-A)^{\frac{1}{2}}\right),
$$

which in particular yields $\left\|(-A)^{-\frac{1}{2}}\right\|=1$.

Let $Z(t, \xi):=\sum_{k=1}^{\infty} \beta_{k} Z_{k}(t) e_{k}(\xi)$, where $\rho<2 q-1-\frac{1}{\alpha}$ and $\left\{Z_{k}(t)\right\}_{k} \geqslant 1$ is a cylindrical $\alpha$-stable process on $H$ with $\left\{Z_{k}(t)\right\}_{k \in \mathbb{N}}$ being i.i.d. one dimensional symmetric $\alpha$-stable process sequence with $1<\alpha<2$. Moreover, there exist some $\mathrm{C}_{1}, \mathrm{C}_{2}>0$ such that for $k \in \mathbb{N}$,

$$
C_{1} \lambda_{k}^{-\beta} \leqslant\left|\beta_{k}\right| \leqslant C_{2} \lambda_{k}^{-\beta} \quad \text { for some } \quad \beta>0 .
$$

For $\mathrm{t} \in[0, \mathrm{~T}]$ and $x \in[0, \pi]$, let

$$
x(t)(x):=u(t, x), \quad g(t, x(t-\tau))(x):=\int_{0}^{\pi} \varphi(-\tau, \zeta, x) u(t-\tau, \zeta) d \zeta
$$

and

$$
f(t, x(t-\tau))(x):=\phi(u(t-\tau), x) .
$$

Then (4.1) can be rewritten in the form (1.1). Then, using $\varphi(\cdot, \cdot, 0)=\varphi(\cdot, \cdot, \pi)=0$, combining (4.2)-(4.4) with the Hölder's inequality, we obtain $\|(-A)^{\frac{1}{2}}\left(g(t, x(t-\tau))-g(t, y(t-\tau))\left\|^{2} \leqslant N\right\| x(t-\tau)-y(t-\tau) \|^{2}\right.$ (see [26]). Hence, (H2) and (H3) hold.

On the other hand, by simple calculation we have

$$
\sum_{k=1}^{\infty} \beta_{k}^{\alpha} \leqslant \sum_{k=1}^{\infty} C_{2}^{\alpha} \lambda_{k}^{-\alpha \beta}=\sum_{k=1}^{\infty} C_{2}^{\alpha} k^{-2 \alpha \beta} .
$$

Thus, we can see that (3.4) holds if $\beta>\frac{1}{2 \alpha}$. Consequently, by Theorem 3.4, there exists a unique mild solution to (4.1) provided that $\beta>\frac{1}{2 \alpha}$ and $\sigma$ satisfies the assumption (H4). 


\section{Conclusion}

In this paper, a class of fractional neutral stochastic partial differential equations driven by $\alpha$-stable process are discussed. By estimating the pth moment of $\alpha$-stable noise and using the Banach fixed-point theorem, the existence and uniqueness of the mild solutions to this kind of equations driven by $\alpha$-stable process are obtained.

\section{Acknowledgment}

This work is supported by Natural Science Foundation of Hubei Province (No. 2016CFB479) and China postdoctoral fund (No. 2017M610216).

\section{References}

[1] D. Applebaum, Lévy processes and stochastic calculus, Second edition, Cambridge Studies in Advanced Mathematics, Cambridge University Press, Cambridge, (2009). 2

[2] D. Baleanu, H. Jafari, H. Khan, S. J. Johnston, Results for mild solution of fractional coupled hybrid boundary value problems, Open Math., 13 (2015), 601-608. 1

[3] J.-H. Bao, C.-G. Yuan, Numerical analysis for neutral SPDEs driven by $\alpha$-stable processes, Infin. Dimens. Anal. Quantum Probab. Relat. Top., 17 (2014), 17 pages. 1

[4] J. Cui, L.-T. Yan, Existence result for fractional neutral stochastic integro-differential equations with infinite delay, J. Phys. A, 44 (2011), 16 pages. 1

[5] G. Da Prato, J. Zabczyk, Stochastic equations in infinite dimensions, Encyclopedia of Mathematics and its Applications, Cambridge University Press, Cambridge, (1992). 1

[6] Z. Dong, L.-H. Xu, X.-C. Zhang, Invariant measures of stochastic 2D Navier-Stokes equation driven by $\alpha$-stable processes, Electron. Commun. Probab., 16 (2011), 678-688. 1

[7] U. Haagerup, The best constants in the Khintchine inequality, Studia Math., 70 (1982), 231-283. 3

[8] R. Jahanipur, Nonlinear functional differential equations of monotone-type in Hilbert spaces, Nonlinear Anal., 72 (2010), 1393-1408. 1

[9] F. Jiao, Y. Zhou, Existence of solutions for a class of fractional boundary value problems via critical point theory, Comput. Math. Appl., 62 (2011), 1181-1199. 1

[10] P. Kalamani, D. Baleanu, S. Selvarasu, M. M. Arjunan, On existence results for impulsive fractional neutral stochastic integro-differential equations with nonlocal and state-dependent delay conditions, Adv. Difference Equ., 163 (2016), 36 pages. 1

[11] M. Kerboua, A. Debbouche, D. Baleanu, Approximate controllability of Sobolev type fractional stochastic nonlocal nonlinear differential equations in Hilbert spaces, Electron. J. Qual. Theory Differ. Equ., 2014 (2014), 16 pages.

[12] K.-X. Li, Stochastic delay fractional evolution equations driven by fractional Brownian motion, Math. Methods Appl. Sci., 38 (2015), 1582-1591.

[13] K.-X. Li, J.-G. Peng, Controllability of fractional neutral stochastic functional differential systems, Z. Angew. Math. Phys., 65 (2014), 941-959. 1

[14] K.-X. Li, J.-G. Peng, J.-H. Gao, Existence results for semilinear fractional differential equations via Kuratowski measure of noncompactness, Fract. Calc. Appl. Anal., 15 (2012), 591-610. 3

[15] K. Liu, Stability of infinite dimensional stochastic differential equations with applications, Chapman \& Hall/CRC Monographs and Surveys in Pure and Applied Mathematics, Chapman \& Hall/CRC, Boca Raton, FL, (2006). 1

[16] C. Lizama, G. M. N'Guérékata, Mild solutions for abstract fractional differential equations, Appl. Anal., 92 (2013), 1731-1754. 1

[17] J.-W. Luo, K. Liu, Stability of infinite dimensional stochastic evolution equations with memory and Markovian jumps, Stochastic Process. Appl., 118 (2008), 864-895. 1

[18] F. Mainardi, Fractional calculus and waves in linear viscoelasticity, An introduction to mathematical models, Imperial College Press, London, (2010). 2, 2

[19] A. Pazy, Semigroups of linear operators and applications to partial differential equations, Applied Mathematical Sciences, Springer-Verlag, New York, (1992). 2

[20] E. Priola, J. Zabczyk, Structural properties of semilinear SPDEs driven by cylindrical stable processes, Probab. Theory Related Fields, 149 (2011), 97-137. 1, 3

[21] R. Sakthivel, P. Revathi, Y. Ren, Existence of solutions for nonlinear fractional stochastic differential equations, Nonlinear Anal., 81 (2013), 70-86. 1

[22] R. Sakthivel, S. Suganya, S. M. Anthoni, Approximate controllability of fractional stochastic evolution equations, Comput. Math. Appl., 63 (2012), 660-668. 1

[23] G. Samorodnitsky, M. S. Taqqu, Stable non-Gaussian random processes, Stochastic models with infinite variance, Stochastic Modeling, Chapman \& Hall, New York, (1994). 3 
[24] K.-I. Sato, Lévy processes and infinitely divisible distributions, Translated from the 1990 Japanese original, Revised by the author, Cambridge Studies in Advanced Mathematics, Cambridge University Press, Cambridge, (1999). 2

[25] L.-H. Xu, Ergodicity of the stochastic real Ginzburg-Landau equation driven by $\alpha$-stable noises, Stochastic Process. Appl., 123 (2013), 3710-3736. 1

[26] Y.-C. Zang, J.-P. Li, Stability in distribution of neutral stochastic partial differential delay equations driven by $\alpha$-stable process, Adv. Difference Equ., 2014 (2014), 16 pages. 4.1

[27] X.-C. Zhang, Derivative formulas and gradient estimates for SDEs driven by $\alpha$-stable processes, Stochastic Process. Appl., 123 (2013), 1213-1228. 1

[28] Y. Zhou, F. Jiao, Existence of mild solutions for fractional neutral evolution equations, Comput. Math. Appl., 59 (2010), 1063-1077. 3

[29] Y. Zhou, F. Jiao, Nonlocal Cauchy problem for fractional evolution equations, Nonlinear Anal. Real World Appl., 11 (2010), 4465-4475. 1, 3 